\title{
REFEREES 2015
}

(C) Italian Society of Surgery (SIC) 2016

The Editors-in-chief of Updates in Surgery would like to thank all reviewers for their hard work and loyalty for 2015 to achieve a high scientific standard for the journal.

Agresta Ferdinando (Italy)

Aigner Felix (Germany)

Aldrighetti Luca (Italy)

Altomare Donato Francesco (Italy)

Ardito Francesco (Italy)

Arezzo Alberto (Italy)

Avenia Nicola (Italy)

Balzano Gianpaolo (Italy)

Belli Giulio (Italy)

Bianchi Paolo Pietro (Italy)

Biondi Antonio (Italy)

Boggi Ugo (Italy)

Bonavina Luigi (Italy)

Borghi Felice (Italy)

Boudjema Karim (France)

Campanelli Giampiero (Italy)

Canonico Silvestro (Italy)

Cavallaro Giuseppe (Italy)

Cimino Matteo (Italy)

Corsaro Antonino (Italy)

De Paolis Paolo (Italy)

Del Fabbro Daniele (Italy)

Del Gaudio Massimo (Italy)

Di Sebastiano Pierluigi (Italy)

Dieter Robert S. (United States)

Ercolani Giorgio (Italy)

Ettorre Giuseppe (Italy)

Falletto Ezio (Italy)

Ferrero Alessandro (Italy)

Filauro Marco (Italy)
Fumagalli Uberto (Italy)

Giuliani Antonio (Italy)

Giuliante Felice (Italy)

Grazi Gian Luca (Italy)

Grosso Giuseppe (Italy)

Gruttadauria Salvatore (Italy)

Guerra Francesco (Italy)

Gulino Ferdinando Antonio (Italy)

Horisberger Karoline (Switzerland)

Hsu Po-Kuei (Taiwan, Republic of China)

Kurihara Hayato (Italy)

Langella Serena (Italy)

Lo Tesoriere Roberto (Italy)

Mandalà Vincenzo (Italy)

Mandhan Parkash (Qatar)

Massani Marco (Italy)

Massmann Alexander (Germany)

Miccoli Paolo (Italy)

Milone Marco (Italy)

Mineccia Michela (Italy)

Mistrangelo Massimiliano (Italy)

Miyazaki Masaru (Japan)

Monaco Guglielmo (Italy)

Morino Mario (Italy)

Muratore Andrea (Italy)

Nessi Franco (Italy)

Pallabazzer Giovanni (Italy)

Parmeggiani Domenico (Italy)

Patriti Alberto (Italy)
Pellino Gianluca (Italy)

Pirozzi Felice (Italy)

Polastri Roberto (Italy)

Ragusa Mario (Italy)

Rapisarda Agnese Maria

Chiara (Italy)

Ratti Francesca (Italy)

Rocco Gaetano (Italy)

Romito Raffaele (Italy)

Rosati Riccardo (Italy)

Roscio Francesco (Italy)

Rosso Edoardo (Italy)

Ruol Alberto (Italy)

Russolillo Nadia (Italy)

Saliva Maurizio (Italy)

Salvador Renato (Italy)

Secchi Mario (Argentina)

Selvaggi Francesco (Italy)

Spinelli Antonino (Italy)

Tanaka Takayuki (Japan)

Terrone Carlo (Italy)

Testini Mario (Italy)

Tolver Mette (Germany)

Tropea Alessandro (Italy)

Ueda Kazuhiro (Japan)

Viganò Luca (Italy)

Zago Mauro (Italy)

Zerbi Alessandro (Italy) 